\title{
APLICAÇÕES DA NANOTECNOLOGIA NO DIAGNÓSTICO E TRATAMENTO DE CÂNCER
}

\author{
FALLEIROS, João Paulo Barbosa ${ }^{1}$ \\ BRANDL, Ana Lúcia ${ }^{2}$ \\ FONSECA, Ana Rita Alvarenga da ${ }^{3}$
}

\begin{abstract}
RESUMO: A nanociência e a nanotecnologia $(\mathrm{N} \& \mathrm{~N})$ tem atraído, nos últimos anos, bastante interesse da comunidade científica, do setor tecnológico e da mídia. Tamanho interesse se deve pelas diferenças apresentadas por esses materiais, em termos de suas propriedades físicas e químicas, quando comparados aos materiais macroscópicos. Uma das aplicações promissoras da $\mathrm{N} \& \mathrm{~N}$ trata-se da área biomédica. Nanopartículas magnéticas biocompatíveis tem possibilitado o desenvolvimento de técnicas para o diagnóstico e o tratamento de câncer, tais como a ressonância magnética nuclear, a vetorização de medicamentos e a hipertermia magnética. É apresentado neste trabalho o estado da arte das principais técnicas utilizadas na terapia oncológica, bem como os desafios e tendências das pesquisas na área.
\end{abstract}

Palavras-chave: Aplicações biomédicas da nanotecnologia. Nanopartículas magnéticas biocompatíveis. Terapia oncológica.

\section{APPLICATIONS OF NANOTECHNOLOGY IN CANCER THERAPY}

SUMMARY: Nanoscience and nanotechnology $(\mathrm{N} \& \mathrm{~N})$ have attracted, in the last years, special attention of the academic community, technology sector and media. This interest can be explained by changes observed in chemical and physical properties of these materials when compared with the bulk ones. One of the promising applications of N\&N comes from the biomedical area. Biocompatible magnetic nanoparticles have enabled the development of techniques for diagnosis and treatment of cancer, such as magnetic resonance imaging (MRI), drug delivery and magnetic hyperthermia. In this paper is presented the state of the art about main techniques used in cancer therapy, challenges and trends of research in the area.

Keywords: Biomedical applications of nanotechnology. Biocompatible magnetic nanoparticles. Cancer therapy.

\section{INTRODUÇÃO}

A nanociência e a nanotecnologia $(\mathrm{N} \& \mathrm{~N})^{4}$ são áreas do conhecimento que tem despertado, nos últimos anos, bastante interesse da comunidade científica, do setor tecnológico e da mídia, tanto devido aos desafios relacionados à compreensão de suas propriedades físicas e químicas, quanto às diversas possibilidades de aplicações tecnológicas

\footnotetext{
${ }^{1}$ Graduação em Engenharia de Produção pela Universidade Federal de São Carlos, campus Sorocaba. Bolsista de Iniciação Científica financiada pelo Conselho Nacional de Desenvolvimento Científico e Tecnológico

${ }^{2}$ Pós-Doutorado em Física pela Universidade de São Paulo, atua na área de magnetismo e propriedades magnéticas de materiais nanoestruturados.

${ }^{3}$ Graduação em farmácia pela Universidade de Uberaba.

${ }^{4} \mathrm{~A} N \& \mathrm{~N}$ lida com fenômenos e materiais em escala nanométrica - um nanômetro equivale a $10^{-9}$ metros, ou um bilionésimo de metro.
} 
que esses materiais apresentam. Os materiais nanométricos, ou nanomateriais, correspondem a filmes finos (uma dimensão nanométrica), nanofios (duas dimensões nanométricas) e nanopartículas (três dimensões nanométricas).

Tamanho interesse no estudo e desenvolvimento destes materiais reside no fato de apresentarem propriedades físicas e químicas diferentes das observadas nos materiais macroscópicos ou massivos (BATLE; LABARTA, 2002). Destaque é dado para as nanopartículas (NPs) magnéticas, que tem sido amplamente estudadas e cujas aplicações estão gerando novas fronteiras para o desenvolvimento tecnológico.

Os sistemas magnéticos nanocristalinos são constituídos de nanopartículas magnéticas que podem estar dispersas em meios sólidos (sólidos granulares) ou em meios líquidos (fluidos magnéticos ou ferrofluidos) (KNOBEL, 2000). No caso dos sólidos granulares, as matrizes nas quais as NPs estão imersas podem ser condutoras ou isolantes, cristalinas ou amorfas. Já no caso dos ferrofluidos, as NPs se encontram num líquido carreador de base aquosa ou oleosa, geralmente recobertas com uma camada orgânica que evita o contato entre as NPs e sua decantação.

A redução drástica nas dimensões dos sistemas nanométricos provoca mudanças em suas propriedades magnéticas. Tais diferenças, em comparação ao comportamento de materiais massivos, se devem fundamentalmente a dois fatores (SKOMSKI, 2003): (i) os tamanhos característicos das nanopartículas (1 100 nm) são comparáveis aos comprimentos de correlação e de troca ${ }^{5}$ e (ii) existe um número relativamente grande de átomos na superfície das nanopartículas.

O estudo dos sistemas magnéticos nanoestruturados é importante tanto do ponto de vista da ciência básica quanto do desenvolvimento de aplicações tecnológicas. Fenômenos físicos interessantes estão presentes nesses sistemas como o superparamagnetismo (BEAN; LIVINGSTON, 1959; CHIEN, 1991), a magneto-resistência gigante (GMR) (XIAO et al., 1993), o efeito Hall gigante (EHE) (PAKHOMOV et al., 1995), a magneto- resistência por tunelamento (TMR) (MITANI et al., 1998) e o comportamento do tipo vidro de spin (spinglass-like) (CHILDRESS; CHIEN, 1991).

Diversas aplicações tecnológicas são intensamente pesquisadas nos últimos anos como, por exemplo, na indústria da informática, novos métodos de armazenamento de dados, leitura e gravação de informação em mídias magnéticas, na produção de imãs permanentes mais fortes e de micro-sensores magnéticos, nas indústrias têxteis e de tintas, dentre várias outras. Uma área de aplicação das NPs magnéticas que merece destaque é a biomedicina, que

\footnotetext{
${ }^{5}$ Os comprimentos de correlação e de troca são os comprimentos característicos de sistemas nanométricos; por exemplo, a distância entre dois átomos em um sítio cristalino corresponde a um comprimento característico de um determinado material cristalino.
} 
tem recebido investimentos massivos e crescentes ao longo dos últimos 50 anos (BARRAT, 2003). O fato de as NPs apresentarem tamanhos comparáveis aos de entidades biológicas, como células, vírus, moléculas, proteínas e até mesmo genes, as torna adequadas às aplicações biomédicas. Tartaj e colaboradores, em 2003, publicaram um artigo apresentando os principais métodos de síntese de NPs magnéticas para aplicações biomédicas (TARTAJ et al., 2003).

Dentre essas aplicações bastante promissoras, pode-se destacar a utilização de NPs magnéticas biocompatíveis para o diagnóstico e tratamento de câncer em técnicas como o aumento do contraste em imagens de ressonância magnética (MRI), a separação celular, a vetorização de medicamentos e a hipertermia magnética (KNOBEL; GOYA, 2004; TARTAJ et al., 2003; PANKHURST et al., 2003). A nanotecnologia dedicada à terapia oncológica permite a detecção rápida e sensível de células malignas em estágios iniciais e seu controle de forma não invasiva.

As aplicações oncológicas das NPs biocompatíveis são baseadas nos seguintes princípios físicos (GOYA et al., 2008): (i) a aplicação de um gradiente de campo magnético permite posicionar remotamente as NPs no interior de órgãos ou tecidos; (ii) a utilização do momento magnético das NPs permite provocar perturbações na ressonância nuclear de prótons e (iii) as perdas de calor das NPs submersas em fluidos magnéticos podem ser aproveitadas para a termocitolise de tumores.

Além disso, a grande superfície relativa das NPs pode ser apropriadamente modificada, ou funcionalizada, para receber agentes biológicos, moléculas orgânicas, vitaminas, antibióticos, peptídeos ou recobrimento polimérico com a finalidade de alterar suas propriedades ou programá-las para se acumularem especificamente em células "alvo". Se unirmos a funcionalização das NPs com as propriedades físicas envolvidas em suas aplicações biomédicas, vemos que tais sistemas apresentam um número enorme de aplicações tanto in vitro como in vivo, diagnósticas ou terapêuticas.

Algumas aplicações das NPs na área biomédica, como a de obtenção de imagens por ressonância magnética, já se encontram em fase avançada de estudo e estão sendo aplicadas (NANZ et al., 2000; SAINI et al., 2000; GALVÃO et al., 2002); outras, entretanto, ainda encontram-se em fase de pesquisa e desenvolvimento, como é o caso da hipertermia magnética e da vetorização de medicamentos ( TARTAJ et al., 2003; BABINCOVÁ et al., 2000). Como envolvem várias áreas do conhecimento, as aplicações biomédicas da nanotecnologia requerem a formação de equipes multidisciplinares para seu desenvolvimento.

Grande esforço tem sido dedicado à formação dessas equipes provenientes de Universidades e Centros de Pesquisa. 
Este artigo tem como principal objetivo apresentar o estado da arte da aplicação da nanotecnologia na biomedicina, em especial para o tratamento do câncer. Para tal, são abordados os princípios físicos envolvidos em suas aplicações, os principais desafios e as perspectivas para esta área relativamente nova da ciência.

\section{NANOMAGNETISMO}

Para entender os fenômenos magnéticos que ocorrem em escala nanométrica, é necessário conhecer alguns conceitos do magnetismo dos materiais. Quando um imã permanente é dividido em duas partes, nenhuma delas perderá seu magnetismo ou tornar-se-á um pólo isolado; ao contrário, cada uma das partes constituirá um novo imã. Se essas partes resultantes fossem novamente divididas, e assim sucessivamente, em pedaços cada vez menores, existiriam minúsculos imãs cujas propriedades seriam as mesmas apresentadas pelo imã original.

Esses minúsculos imãs, ou imãs elementares, foram, a princípio, denominados imãs moleculares ou atômicos e, posteriormente, dipolos magnéticos. O dipolo é a menor unidade fundamental do magnetismo, pois, não há como dividi-lo em pólos separados e não existe um pólo com carga isolada.

Quando um campo magnético externo $H$ é aplicado a um material, ocorre uma mudança em seus dipolos magnéticos originando uma resposta macroscópica: a magnetização $M$ (ou momento magnético por unidade de volume). O momento magnético, ou $\mu$, diz respeito ao momento do torque exercido sobre um dipolo quando este se encontra sobre a aplicação de um campo magnético externo (CULLITY, 1972).

De acordo com Faria e Lima (2005), os materiais podem ser separados em classes de acordo com a orientação de seus momentos magnéticos em relação à aplicação de um campo magnético externo. São cinco as classes em que os materiais podem ser classificados: diamagnéticos, paramagnéticos, ferromagnéticos, antiferromagnéticos e ferrimagnéticos.

O diamagnetismo é um fenômeno que ocorre em todos os materiais, mas frequentemente tem seu efeito encoberto por outros mais fortes, como o paramagnetismo e o ferromagnetismo. O diamagnetismo nada mais é do que a manifestação microscópica da Lei de Lenz (FARIA; LIMA, 2005), que diz que uma variação no fluxo magnético dá origem a uma corrente induzida no material, e gera um fluxo magnético oposto ao inicial.

Substâncias diamagnéticas não apresentam, portanto, um momento magnético externamente, ou seja, pólos magnéticos como um imã permanente; mas, quando submetidas a um campo externo, respondem com uma tendência a se afastarem da região em que a 
intensidade do campo é maior. Por esse motivo diz-se que possuem um magnetismo negativo, pois quando na presença de um campo, produzem seu próprio campo magnético, mas com polaridade oposta ao campo externo.

Quando o átomo contém um número ímpar de elétrons, existirá um momento magnético resultante devido ao elétron não emparelhado, que dá origem ao paramagnetismo (FARIA; LIMA, 2005). No paramagnetismo, os momentos magnéticos atômicos existem mesmo na ausência de um campo magnético externo. Quando um campo é aplicado, os momentos magnéticos atômicos se orientam em sua direção e produzem um campo externo próprio, resultante do alinhamento dos dipolos elementares atômicos.

Os materiais ferromagnéticos, antiferromagnéticos e ferrimagnéticos são caracterizados pelo ordenamento espontâneo de seus momentos magnéticos. No ferromagnetismo, os momentos se ordenam paralelamente, resultando em uma magnetização não nula mesmo na ausência de um campo aplicado. No antiferrimagnetismo, existe uma interação de troca entre átomos vizinhos que força os momentos magnéticos a assumirem orientações antiparalelas e, portanto, os materiais com este comportamento apresentam um magnetismo externo muito pequeno ou mesmo nulo (FARIA; LIMA, 2005).

No ferrimagnetismo, por fim, o alinhamento dos momentos magnéticos é principalmente antiparalelo, contudo, como os momentos magnéticos vizinhos possuem magnitudes distintas, a magnetização resultante não é nula, como nos materiais antiferromagnéticos.

O nanomagnetismo é a área da física que estuda as propriedades magnéticas de materiais que se apresentam em escala nanométrica (um nanômetro equivale a $10^{-9}$ metros) ou mesoscópica. O nanomagnetismo estuda as propriedades e as aplicações do magnetismo em partículas isoladas (nanopartículas), nanofios, filmes finos e multicamadas, além de amostras volumosas que incorporam partículas nanoscópicas (materiais nanoestruturados).

A redução do tamanho característico das partículas traz diversas mudanças às suas propriedades físicas (PELECKY; RIEKE, 1996). Uma das mudanças mais marcantes referese ao regime magnético que esses sistemas podem apresentar, chamado de regime superparamagnético (SPM). Com o volume reduzido das NPs, a energia térmica $k_{B} T$ passa a ser de magnitude comparável a energia de anisotropia ${ }^{6}$ da partícula, que é dada por $K V$, sendo $K$ uma constante de anisotropia uniaxial efetiva, $V$ o volume da NP, $T$ a temperatura do sistema e $k_{B}$ a constante de Boltzmann. Isto significa que as flutuações térmicas no sistema

\footnotetext{
${ }^{6}$ Denomina-se anisotropia magnética a preferência que um material magnético possui para se magnetizar em determinada direção. A anisotropia magnética é importante em grãos monodomínios, pois a maior ou menor dificuldade de magnetização depende diretamente da medida dessa propriedade (GUIMARÃES, 2000).
} 
são suficientes para que o momento magnético da NP reverta de uma posição de equilíbrio para outra, conforme ilustrado na figura 1(a).

Considerando um sistema monodisperso (um único tamanho de partículas) e nãointeragente, a taxa com que ocorrem as transições de um mínimo de energia a outro depende do fator de Boltzmann para a energia de anisotropia efetiva $K V$ e a energia térmica $k_{B} T$ e de uma constante de tempo $\tau_{0}$ (DORMAN et al., 1997), que caracteriza a frequiência de tentativas de salto à barreira de anisotropia, seguindo uma lei do tipo Arrhenius (NÉEL, 1949):

$$
\tau=\tau_{\mathrm{o}} \exp \left(\frac{E_{a}}{E_{T}}\right)=\tau_{\mathrm{o}} \exp \left(\frac{K V}{k_{B} T}\right)
$$

Se o tempo de uma dada medida experimental é maior que o tempo de relaxação do sistema estudado, dado pela equação 1, observam-se diversas transições entre os dois estados de equilíbrio. Neste caso, diz-se que o sistema está no estado superparamagnético, pois seu comportamento é análogo ao de um paramagneto clássico, com a diferença que aqui o valor do momento magnético é muito maior que 1 ou $2 \mathrm{k}_{\mathrm{B}}$ típicos de um átomo e sim tem valores da ordem de $10000 \mathrm{k}_{\mathrm{B}}$, daí a origem do termo superparamagnético.

Se, por outro lado, temos um tempo de medida menor que o tempo de relaxação do sistema estudado, isso significa que, durante nossa medição experimental, o momento magnético da partícula ficará fixo, ou como é mais habitual dizer, "bloqueado", em um mínimo de energia. Nesse caso, diz-se que o sistema está no estado bloqueado, pois não se observam transições por sobre a barreira de energia de anisotropia durante o tempo de observação experimental. É interessante salientar que mudando o tempo característico de uma medida experimental, um sistema pode deixar de ser bloqueado e passar a ser SPM, ou seja, a determinação do estado do sistema depende fundamentalmente do tempo de medida.

Para ilustrar a diferença entre o comportamento magnético nesses dois estados, superparamagnético e bloqueado, a figura 1(b) mostra duas curvas de magnetização em função do campo magnético aplicado $(M \times H)$ para uma amostra de um filme granular de partículas de cobalto imersas numa matriz isolante de óxido de silício. Uma das curvas foi medida a temperatura de $5 \mathrm{~K}$, quando o sistema está no estado bloqueado (temos coercividade e remanência não nulas), e outra a $300 \mathrm{~K}$, quando o sistema está no estado superparamagnético (curva anisterética, como num paramagneto).

Podemos notar que existe uma temperatura que separa os dois regimes magnéticos, conhecida como temperatura de bloqueio $\left(T_{B}\right)$. Esta temperatura pode ser estimada a partir da equação 1, igualando o tempo de relaxação $\tau$ a um tempo de medida $t_{m}$ típico de medidas de 
magnetização, 100 segundos, por exemplo, de onde obtemos a expressão $T_{B}=K V / 25 k_{B}$, que depende do volume e da constante de anisotropia das NPs.
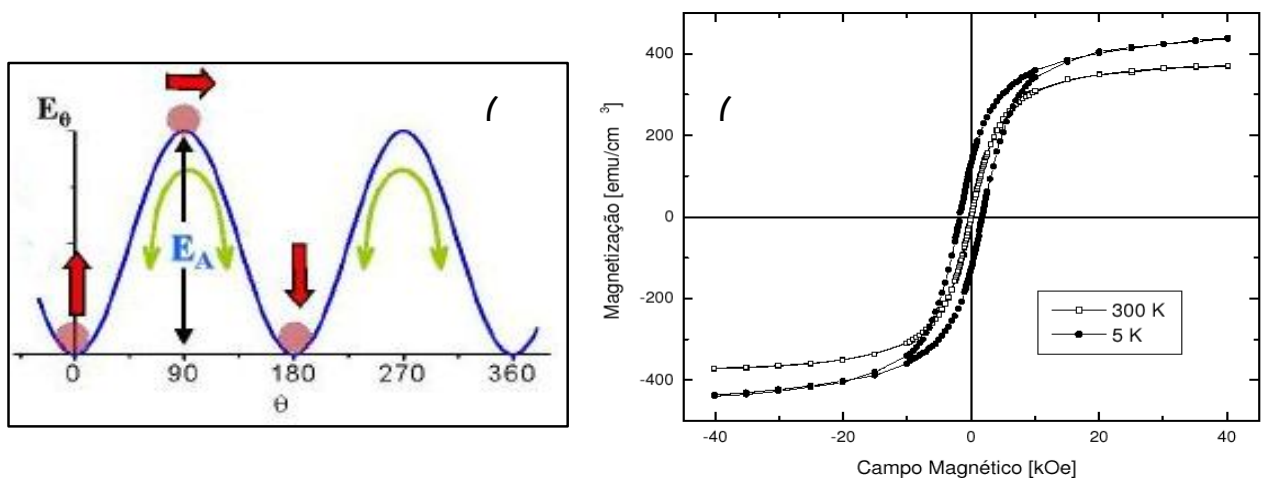

Figura 1: (a) Perfil da energia de anisotropia uniaxial efetiva, ilustrando a barreira $E_{A}=K V$ que o momento magnético da NP deve ultrapassar para passar de uma posição de equilíbrio a outra; (b) Curvas de magnetização em função do campo magnético aplicado para uma amostra de $\mathrm{Co}_{0.35}\left(\mathrm{SiO}_{2}\right)_{0.65}$, depositada em substrato de kapton. As curvas foram medidas em duas temperaturas diferentes no magnetômetro SQUID do LMBT/IFGW/UNICAMP, com campos de até $40 \mathrm{kOe}$, com campo magnético aplicado longitudinalmente ao plano do filme granular.

Considerando um conjunto de nanopartículas monodomínio, não-interagentes, com momento magnético uniformemente distribuído $\mu$ e com constante de anisotropia desprezível $(K \approx 0$ ), é possível calcular sua magnetização através da função de Langevin (CULLITY, 1972). Considerando a presença de uma distribuição de tamanhos, o que ocorre na maioria das amostras reais, a magnetização pode ser obtida fazendo a convolução da função de Langevin com uma função de distribuição apropriada $f(\mu)$ (lognormal ou gaussiana, mais comumente):

$$
M(H, T)=\int_{0}^{\infty} \mu L\left(\frac{\mu H}{k_{B} T}\right) f(u) d \mu
$$

onde $H$ é o campo magnético aplicado, $N$ é o número de partículas por unidade de volume e $L$ é a função de Langevin. Analisando a expressão acima, notamos que as curvas de magnetização $M(H, T)$ medidas em diferentes temperaturas devem se sobrepor quando graficadas como uma função de $H / T$, sendo essa característica conhecida como a lei de escala clássica do superparamagnetismo (CULITY, 1972).

Dessa forma, temos que duas assinaturas fundamentais de um superparamagneto são: cumprimento da lei de escala clássica e ausência de remanência e coercividade na curva $M(H)$. Essa situação somente ocorre se temos um sistema magneticamente diluído (interações magnéticas desprezíveis) e se estamos numa temperatura bem acima da temperatura de bloqueio média do sistema $\left\langle T_{B}\right\rangle$, onde nem mesmo as partículas de maior volume se encontram no regime bloqueado. 


\section{APLICAÇÕES BIOMÉDICAS}

As técnicas que utilizam NPs magnéticas na terapia oncológica podem ser usadas dentro (in vivo) ou fora (in vitro) do organismo. As aplicações in vivo podem ser divididas em diagnósticas (imagens de ressonância magnética) ou terapêuticas (marcação celular, vetorização de medicamentos e hipertermia magnética); enquanto as aplicações in vitro são apenas diagnósticas (separação e seleção). Serão apresentadas neste trabalho as principais aplicações in vivo que estão sendo utilizadas e que estão em fase de pesquisa na terapia oncológica. Para maiores informações sobre as aplicações in vitro, os seguintes trabalhos são sugeridos: Pankhurst e colaboradores (2003), Goya e colaboradores (2008) e Mrinmoy e colaboradores (2008).

\section{RESSONÂNCIA MAGNÉTICA NUCLEAR}

A ressonância magnética nuclear tem assumido grande importância em relação a outras técnicas de obtenção de imagens. Trata-se de uma técnica não invasiva e não destrutiva capaz de obter imagens em duas ou três dimensões ideais para a visualização de tecidos moles, mostrando anatomia e fisiologia simultaneamente, utilizada para diagnósticos, exames pré-cirúrgicos e monitoramento de terapias, sem limitação de volume ou profundidade do tecido analisado (BULTE et al., 2002).

O desenvolvimento de scanners e receptores mais sofisticados para os dispositivos de obtenção de imagens por ressonância magnética (MRI) tem provido uma redução no tempo de obtenção das imagens, que da escala de horas passou para a de segundos (ZHANG; WEBB, 2005), além do aumento da resolução desses aparelhos, da escala de centímetros para a de micrometros (JOHNSON et al., 1993) resultando na obtenção de imagens microscópicas de tecidos in vivo. Esses avanços tornaram a técnica de obtenção de imagens por ressonância magnética uma poderosa ferramenta para o diagnóstico e tratamento oncológico.

A MRI baseia-se no contrabalanço entre o pequeno momento magnético $\mu \mathrm{em}$ um próton (spin) e o grande número de prótons presentes nos átomos de hidrogênio dos tecidos biológicos (rede), o que leva a um efeito mensurável de prótons alinhados na presença de altos campos magnéticos estáticos $B_{0}$ (que variam de 1 a 2 Tesla) (PANKHURST et al., 2003). O sinal pode ser então capturado por meio da absorção ressonante: aplicando-se um campo magnético variável no tempo (5-100 MHz) em uma direção perpendicular a $B_{0}$, a frequência de precessão de Larmor dos prótons muda de acordo com a expressão $\omega_{0}=\gamma B_{0}$, onde $\gamma$ representa a razão giromagnética dos prótons. 
Para prótons referentes aos núcleos de hidrogênio nas moléculas de água $\left({ }^{1} \mathrm{H}\right)$, abundantes nos tecidos vivos, a razão giromagnética vale $\gamma=2,67.10^{8} \mathrm{rad} \mathrm{s}^{-1} \mathrm{~T}^{-1}$. Portanto, sob um campo magnético $B_{0}$ de intensidade igual a $1 \mathrm{~T}$, a frequência de precessão de Larmor corresponde ao campo de rádio-frequência $\frac{\omega_{0}}{2 \pi}=42,57 \mathrm{MHz}$.

Para se obter uma resposta coerente do momento magnético líquido $m$ dos prótons no scanner de MRI, na prática, o campo transversal de rádio-frequência é aplicado em uma sequência pulsada. A partir do momento em que o pulso de rário-frequência é desligado, a relaxação da resposta coerente é medida através das correntes induzidas nas bobinas pick-up do scanner. Para um campo magnético aplicado paralelamente ao eixo $z$, os sinais de relaxação são da forma (PANKHURST et al., 2003):

$$
\begin{aligned}
& m_{z}=m\left(1-e^{-t / T_{1}}\right) \\
& m_{x, y}=m \operatorname{sen}\left(\omega_{0} t+\emptyset\right) e^{-t} / T_{z}
\end{aligned}
$$

Nas equações acima, $T_{1}$ e $T_{2}$ são os tempos de relaxação longitudinal (spin-rede) e transversal (spin-spin), respectivamente, e $\emptyset$ é a constante de fase. A relaxação longitudinal reflete a perda de energia (na forma de calor) do sistema para sua rede vizinha, e é a medida do acoplamento dipolar dos momentos dos prótons à suas vizinhanças. A relaxação no plano $x y$ é relativamente rápida, e é conduzida pela perda de coerência de fase nos prótons precessantes devido às interações magnéticas entre eles e com outros momentos flutuantes do tecido vivo.

A defasagem pode ser também afetada por não homogeneidades no campo longitudinal aplicado, levando à substituição de $T_{2}$ na equação 5 pelo tempo de relaxação mais curto, $T_{2}^{*}$ (PANKHURST et al., 2003):

$$
\frac{1}{T_{2}^{*}}=\frac{1}{T_{2}}+\gamma \frac{\Delta B_{0}}{2}
$$

onde $\Delta B_{0}$ é a variação no campo magnético devido a distorções na homogeneidade do campo aplicado ou variações locais na susceptibilidade magnética do sistema.

Quando nanopartículas superparamagnéticas são usadas como agentes de contraste para a obtenção de imagens por ressonância magnética, elas estabelecem um campo dipolar perturbador substancial localizado que leva a uma redução em $T_{z}^{*}$, juntamente com uma menos notável redução em $T_{1}$, gerando um contraste negativo que aumenta os detalhes e contornos da imagem. 
Estudos sobre NPs superparamagnéticas utilizadas como agentes de contraste para MRI vem sendo feitos há mais de 20 anos. Em 1986, Saini e colaboradores reportaram a utilização de um óxido de ferro (magnetita) para contraste em imagens obtidas do fígado de ratos (SAINI et al., 1986); contudo, observou-se efeitos de aglomeração entre elas, o que fazia com que fossem rapidamente absorvidas pelo sistema reticuloendotelial e excretadas do organismo. Em 1989, Pouliquen e colaboradores apresentaram então um agente para contraste de MRI constituído de partículas superparamagnéticas de óxido de ferro encapsuladas em micro cápsulas biodegradáveis (POULIQUEN et al., 1989). O recobrimento polimérico das NPs reduziu os efeitos de aglomeração entre as partículas permitindo que as imagens fossem obtidas com a injeção de uma dosagem menor do ferrofluido magnético melhorando, assim, a resolução e nitidez das imagens.

As nanopartículas biocompatíveis são seletivamente absorvidas pelo sistema reticuloendotelial, uma rede de células que revestem os vasos sanguíneos cuja função é remover substâncias estranhas da corrente sanguínea (LAWACZECK et al., 1997). As NPs são então excretadas pelo fígado. Partículas cujo diâmetro ultrapassa os $30 \mathrm{~nm}$ são, segundo Weissleder e colaboradores (1990), mais rapidamente eliminadas via baço e fígado. Por outro lado, partículas menores, com diâmetro de até $10 \mathrm{~nm}$, não são facilmente reconhecidas pelo sistema reticuloendotelial.

Quanto menor a partícula, portanto, maior será sua meia-vida na corrente sanguínea (RUEHM et al., 2001), possibilitando assim que imagens de MRI sejam obtidas em um intervalo de tempo maior entre a aplicação do agente de contraste e a obtenção das imagens de MRI, conforme apresentam Dosset e colaboradores (1999). Além disso, partículas com diâmetros menores do que $10 \mathrm{~nm}$ destacam ainda mais o comportamento do próton de diferentes tecidos, permitindo avanços na técnica de MRI, como obtenção de imagens do sistema vascular (WACKER et al., 2003) e nervoso (DOSSET et al., 1999).

Atualmente, os agentes de contraste encontrados no mercado ${ }^{7}$ são constituídos por nanopartículas superparamagnéticas de óxidos de ferro ${ }^{8}$ apresentando tamanhos de 5 a 10 nanômetros, recobertas com um polissacarídeo, como o dextran, para propósito de redução da aglomeração entre as partículas e sua estabilização em soluções. Outros ligantes são utilizados para a funcionalização das NPs, principalmente moléculas orgânicas de cadeia longa. A quitosana, que já é utilizada em várias aplicações biomédicas, também tem sido bastante usada para o recobrimento das NPs (SILVA et al., 2006).

\footnotetext{
${ }^{7}$ Agentes de contraste constituídos por NPs magnéticas de óxidos de ferro encontrados no mercado são Endorem $^{\circledR}$, Fedirex $^{\circledR}$, Lumirem $^{\circledR}$, Resovist $^{\circledR}$, Sinerem $^{\circledR}$ e Combidex ${ }^{\circledR}$.

${ }^{8}$ Os óxidos de ferro mais utilizados são a magnetita, de fórmula $\mathrm{Fe}_{3} \mathrm{O}_{4}$ e a maghemita, $\gamma-\mathrm{Fe}_{2} \mathrm{O}_{3}$. 
Um caso especial de obtenção de imagens por ressonância magnética nuclear é obtido pela associação de nanopartículas magnéticas a anticorpos monoclonais. Essa associação permite a detecção precoce de micro-metástases e já foi usada com sucesso em tumores malignos nos nódulos linfáticos (MICHEL et al., 2002), fígado (SEMELKA; HELMBERGER, 2001) e cérebro (ENOCHS et al., 1999). Uma nova técnica tem permitido obter imagens de altas resoluções, favorecendo a visualização de tecidos e entidades com poucos micrômetros de espessura. Neste caso, NPs magnéticas são funcionalizadas para se acumularem em uma entidade, um órgão ou um tecido "alvo". A aglomeração das partículas em torno do "alvo" gera um gradiente de campo magnético que permite a visualização, por exemplo, apenas de células "marcadas" - que podem ser as células cancerígenas. Essa nova técnica para obtenção de imagens por ressonância magnética é detalhada por Goya e colaboradores (2008).

Pesquisas atuais na área de ressonância magnética nuclear para a terapia oncológica convergem para (i) a otimização das propriedades magnéticas dos fluidos de contraste e o estudo das interações entre as NPs e o fluido carreador, e do fluido de contraste com o organismo, a fim de reduzir as quantidades aplicadas (dosagens) para a obtenção de imagens cada vez mais nítidas e detalhadas e (ii) o estudo dos efeitos da funcionalização das NPs magnéticas com a finalidade de aprimorá-las para que atinjam especificamente as entidades “alvo", como células, tecidos ou órgãos doentes.

\section{VETORIZAÇÃO DE MEDICAMENTOS}

Dificilmente um fármaco, veiculado em forma farmacêutica convencional, consegue atingir um alvo específico do organismo com concentração adequada para promover o desejado efeito terapêutico. Isso ocorre devido a obstáculos de diversas naturezas ( tais como anatômicos, químicos ou biológicos), interpostos entre o local de administração e o órgão ou tecido "alvo", que necessitam ser vencidos para obtenção do efeito desejado. Oliveira e colaboradores (2004) afirmam que tais limitações tem sido o principal motivo para o desenvolvimento de novas estratégias para a otimização da ação dos medicamentos.

A vetorização de medicamentos, conhecida na literatura científica por drug delivery ou drug targeting, é uma das técnicas nanomagnéticas utilizadas para otimizar a liberação controlada de fármacos sítios-direcionados (SPEISER, 1991). Essa técnica permite a liberação de drogas ou medicamentos na dosagem correta e durante certo período de tempo, diretamente em um tecido ou célula "alvo".

A vetorização ou o direcionamento da substância farmacológica ao sítio de ação 
desejado pode não somente melhorar a eficiência terapêutica, como também permitir a redução da quantidade de droga a ser administrada para obtenção da resposta biológica, minimizando assim os efeitos colaterais indesejáveis (MAGENHEIM; BENITA, 1991).

A liberação sítio-direcionada de fármacos vem sendo estudada na terapia oncológica por se tratar de uma técnica menos agressiva do que os métodos convencionais utilizados atualmente, como a quimioterapia e a radioterapia. Além disso, é possível controlar a dosagem e, dependendo do carreador (ferrofluido ou fluido magnético) utilizado, o tempo entre as liberações de fármaco feitas diretamente nas células cancerígenas, evitando a sobrecarga do organismo com doses massivas e a ação desses medicamentos em células ou tecidos sadios.

Puisieux e Roblot-Treupel (1988) dividem os vetores ${ }^{9}$ em três grupos principais, dependendo de sua composição, suas características e de sua ação no organismo, como vetores de primeira, segunda e terceira geração. Os mesmos autores classificam as NPs magnéticas em vetores do segundo grupo, ou vetores de segunda geração (vetores coloidais ativos). Os vetores de segunda geração ativos são aqueles capazes de veicular o fármaco até o alvo visado, com tamanhos menores que um micrometro e que podem ser administrados por via geral.

Diversos sistemas nanoestruturados estão sendo usados atualmente para a vetorização de medicamentos (KAWAGUCHI, 2000; HAREESH et al., 2010); dentre eles, aqueles baseados em nanopartículas magnéticas destacam-se em virtude de sua capacidade de serem conduzidos e retidos em uma região específica (órgão ou tecido) do corpo humano por meio da aplicação de um campo magnético externo (LÜBBE et al., 2001).

Mais recentemente, pesquisas tem convergido para o desenvolvimento de NPs magnéticas associadas à outra molécula (podendo ser anticorpo, proteína, lectina, hormônio, dentre outros), com o objetivo de aumentar sua especificidade, capazes de reconhecer e se ligarem designadamente à célula, tecido ou órgão "alvo". Os vetores passam a ser classificados então como vetores de terceira geração (PUISIEUX; ROBLOT-TREUPEL, 1988). Os vetores de terceira geração são capazes de reconhecer especificamente o alvo visado e podem ser obtidos por vetores de segunda geração ligados a outras moléculas capazes de dirigi-los aos sítios "alvo".

Tanto os vetores de segunda como os de terceira geração apresentam comportamentos in vivo semelhantes, quando administrados por via intravenosa. Deste modo, os vetores são captados pelas células do sistema reticuloendotelial, especialmente pelos macrófagos do

\footnotetext{
${ }^{9}$ Denominam-se vetores os mecanismos utilizados para o direcionamento sítio-direcionado de medicamentos. São exemplos de vetores: lipossomas, nanocápsulas, nanopartículas, dentre outros.
} 
fígado e pâncreas. Os vetores são incapazes, então, de atravessar a parede dos vasos sanguíneos, salvo possuam endotélio descontinuo ou sinusoidal.

Tais características limitam as potencialidades da utilização de vetores de terceira geração, que geralmente são eliminados pelo organismo antes de atingirem o "alvo". Por conseguinte, é importante que a célula, tecido ou órgão visado seja acessível ao vetor utilizado após sua administração. Células possíveis são, por exemplo, células cancerosas intravasculares (por via intravenal) ou intracavitárias (vias intraperitonial ou intrapleural).

Os desafios desta técnica para o tratamento oncológico residem no desenvolvimento de moléculas direcionadoras mais especificas, capazes de acoplarem-se apenas às células "alvo" sem serem antes eliminadas pelo sistema de defesa do organismo. Além disso, outra questão importante é a forma como a substância ou o medicamento é acoplado à nanopartícula sem que altere suas propriedades magnéticas e de biocompatibilidade. Um resumo dos avanços obtidos no recobrimento e funcionalização de NPs é apresentado por Goya e colaboradores (2008).

\section{HIPERTERMIA MAGNÉTICA}

Denomina-se hipertermia o procedimento terapêutico utilizado para proporcionar aumento de temperatura em uma região (tecido ou órgão) do corpo humano que esteja afetada por uma neoplasia ${ }^{10}$, com o intuito de causar a lise ${ }^{11}$ das células cancerosas. $O$ funcionamento da técnica de hipertermia baseia-se no fato de que temperaturas acima de $41^{\circ} \mathrm{C}$ ( geralmente, de $41^{\circ} \mathrm{C}$ a $46^{\circ} \mathrm{C}$ ) tem o efeito de causar a lise das células cancerosas, uma vez que estas são menos resistentes a aumentos de temperatura do que as células sadias circunvizinhas (TARTAJ et al., 2003).

A hipertermia magnética ou magnetohipertermia é assim chamada quando nanopartículas magnéticas são empregadas no procedimento terapêutico. O método envolve a geração de calor por nanopartículas ferromagnéticas ou superparamagnéticas por meio da aplicação externa de um campo magnético de corrente alternada (AC).

Os mecanismos físicos envolvidos no processo de hipertermia magnética estão relacionados às perdas de energia em forma de calor pelas nanopartículas. Entre eles, as perdas por relaxação magnética e perdas histeréticas, cuja intensidade depende da freqüência do campo magnético aplicado, assim como da natureza das partículas ( características e

\footnotetext{
${ }^{10}$ Neoplasia é o termo utilizado para designar alterações celulares que acarretam um crescimento exagerado das células. A neoplasia pode ser maligna ou benigna e é provocada por mudanças nos genes que regulam o crescimento e a diferenciação celulares.

${ }^{11}$ Lise é o processo de ruptura ou dissolução da membrana plasmática que leva à morte da célula.
} 
propriedades) e de seu recobrimento (funcionalização) (SHINKAI et al., 1994; JÓZEFCZAK; SKUMIEL, 2007). A geração de calor $(P$ ) por partículas ferromagnéticas (micropartículas ou partículas multidomínios) depende de suas perdas histeréticas, cuja relação pode ser descrita por (HERGT et al., 1998):

$$
P=f \Delta U
$$

onde $f$ é a frequiência utilizada e $\Delta U$ é a área do loop histerético provocado pelo campo magnético aplicado.

Em partículas superparamagnéticas (nanopartículas ou partículas monodomínios) a geração de calor $(P)$ se deve às perdas por relaxação magnética (relaxação de Néel ou Brown), que é aproximada por (ROSENSWEIG, 2002):

$$
P=\pi \mu_{0} x f H^{2}
$$

onde $f$ é a frequiência utilizada, $H$ é a intensidade do campo magnético aplicado, $x$ é a componente imaginária da susceptibilidade magnética das partículas e $\mu_{0}$ é a permeabilidade magnética do vácuo.

O uso de nanopartículas superparamagnéticas é mais vantajoso em termos energéticos do que o de partículas ferromagnéticas. As NPs superparamagnéticas são preferidas porque respondem de forma mais eficiente a campos magnéticos externos (devido à propriedade do superparamagnetismo) absorvendo desses mais energia. Em contrapartida, por apresentarem tamanhos muito pequenos, as NPs são facilmente carregadas pelo fluxo sanguíneo e dispersas para outros órgãos (não desejáveis) depois de um curto período de tratamento por hipertermia (MITSUMORI et al., 1996; MITSUMORI et al., 2001 ).

As técnicas apresentadas anteriormente, de ressonância magnética nuclear e de vetorização de medicamentos, são utilizadas em conjunto no tratamento por hipertermia magnética. No processo de hipertermia, NPs são associadas a moléculas específicas, como anticorpos monoclonais, que são específicos para proteínas da membrana de células tumorais, ou células "alvo". Quando administrado, o sistema sítio-direcionado encontra as células “alvo" e permite a obtenção de imagens de ressonância magnética com alta qualidade (potencializadas pelo efeito das NPs superparamagnéticas), possibilitando que metástases sejam diagnosticadas de forma mais eficiente e precoce (SCHÜTT et al., 1995).

Após diagnóstico, as NPs magnéticas absorvidas pelas células cancerosas são então submetidas à aplicação de um campo magnético de corrente alternada (AC) resultando na elevação de sua temperatura (BACRI et al., 1997) e conseqüente lise das células tumorais. Magnetolipossomos e fluidos magnéticos são usados como mediadores no tratamento por hipertermia (SHUBAYEV et al., 2009). Os mediadores podem ser aplicados de forma direta 
no tecido ou órgão em que reside o tumor ou ainda podem ser movimentados até eles por meio da aplicação de um campo magnético externo ao organismo.

Estudos recentes sobre hipertermia magnética focam-se no desenvolvimento de sistemas de hipertermia intracelulares (LI et al., 2010). Nesses sistemas, NPs superparamagnéticas (com tamanho aproximado de $10 \mathrm{~nm}$ ) são geralmente recobertas com uma camada de dextran ou acido oléico com a finalidade de proporcionar estabilidade a fluidos magnéticos, e/ou funcionalizadas com polímeros ou lipossomas para serem ligadas a medicamentos e usados como vetorizadores (ITO et al., 2003; MITSUMORI et al., 2001). A utilização da hipertermia magnética in vivo já é feita há mais de dez anos (YANASE et al., 1998), sendo utilizada como um tratamento complementar a outras terapias oncológicas.

\section{CONSIDERAÇÕES FINAIS}

Nanopartículas magnéticas biocompatíveis podem ser usadas eficazmente no diagnóstico e tratamento de câncer. Esses sistemas podem ser controlados via aplicação de um campo magnético externo. Quando funcionalizadas, as nanopartículas magnéticas podem ser usadas para a vetorização ou direcionamento sitio-direcionado de medicamentos e para a melhoria das propriedades de imagens por ressonância magnética nuclear. Ambas as técnicas citadas são utilizadas também na hipertermia magnética, que tem permitido o tratamento não invasivo e não destrutivo de tecidos e órgãos cancerosos.

A vantagem da utilização de nanopartículas magnéticas no tratamento de câncer está relacionada ao seu nível de alcance intracelular, a redução das dosagens necessárias e ao fato de que esses sistemas atingem unicamente células cancerosas, poupando as células sadias. Muito estudo tem sido realizado nessa área, principalmente ao longo dos últimos 15 anos; contudo, para que se tornem técnicas rotineiras no tratamento de câncer ainda há muito a ser pesquisado. Por se tratar de uma área multidisciplinar e envolver diversos conceitos, o entendimento e a modelagem dos fenômenos físicos, químicos e biológicos relacionados à $\mathrm{N} \& \mathrm{~N}$ tem sido um desafio para a comunidade cientifica.

Diante das aplicações clínicas apresentadas, os sistemas nanométricos devem apresentar baixa toxicidade e um momento magnético de saturação elevado, com a finalidade de minimizar as dosagens necessárias. Neste contexto, a magnetita $\left(\mathrm{Fe}_{3} \mathrm{O}_{4}\right)$ se oferece como um candidato promissor para essas aplicações, visto que possui um baixo grau de toxicidade, permite sua funcionalização com diversos tipos de ligantes e apresenta as propriedades magnéticas requeridas para as técnicas expostas. 


\section{AGRADECIMENTO}

Agradecemos ao Conselho Nacional de Desenvolvimento Científico e Tecnológico (CNPq) e à Universidade Federal de São Carlos (UFSCar) pelo financiamento da pesquisa.

\section{REFERÊNCIAS}

BABINCOVA, M.et al. Blood-specific whole-body electromagnetic hyperthermia. Medical Hypotheses, 55, p. 459-460, 2000.

BACRI, J. C. et al. Use of magnetic nanoparticles for thermolysis of cells in a ferrofluid. In: Scientific and clinical applications of magnetic carriers: an overview. Nova Iorque: Plenum, 1997.

BARRAT, G. Colloidal drug carriers: achievements and perspectives. Cellular and Molecular Life Sciences, 60, p. 21-37, 2003.

BATLLE, X.; LABARTA, A. Finite-size effects in fine particles: magnetic and transport properties. Journal of Physics: Condensed Matter, 35, p. R15-R42, 2002.

BEAN, C. P.; LIVINGSTON, J. D. Superparamagnetism. Journal of Applied Physics, 30, p. 120S-129S, 1959.

BULTE, J. W. M.; DUNCAN, I. D.; FRANK, J. A. In vivo magnetic resonance tracking of magnetically labeled cells after transplantation. Journal of Cerebral Blood Flow and Metabolism, 22, p. 899-907, 2002.

CHIEN, G. L. Granular magnetic solids (invited). Journal of Applied Physics, 69, p. 52675272, 1991.

CHILDRESS, J. R.; CHIEN, C. L. Reentrant magnetic behavior in fcc Co-Cu alloys. Physics Review B, 43, p. 8089-8093, 1991.

CULLITY, B. D. Introduction to Magnetic Materials. Addison-Wesley, Reading, 1972.

DORMANN, J. L.; FIORANI, D.; TRONC, E. Magnetic Relaxation in Fine-Particle Systems. Advances in Chemical Physics, 98, 1997.

DOSSET, V.et al. Dose and scanning delay using USPIO for central nervous system macrophage imaging. Magnetic Resonance Materials in Physics, Biology and Medicine, 8, p. 185-189, 1999.

ENOCHS, W. S.et al. Improved delineation of human brain tumors on MR images using a long-circulating superparamagnetic iron oxide agent. Journal of Magnetic Resonance Imaging, 9, p. 228-232, 1999.

FARIA, R. N.; LIMA, L. F. C. P. Introdução ao magnetismo dos materiais. São Paulo: Livraria da Física, 2005. 
GALVÃO, M. M. F.et al. Uso do contraste oral negativo em exames de colangiografia por ressonância magnética. Radiologia Brasileira, v. 35, n.5, p. 267-271, 2002.

GOYA, G. F.; GRAZÚ, V.; IBARRA, M. R. Magnetic nanoparticles for câncer therapy. Current Nanoscience, 4, p. 1-16, 2008.

GUIMARÃES, A. P. Propriedades magnéticas de sistemas granulares. Revista Brasileira do Ensino de Física, 22, p. 382-386. 2000.

HAREESH, B. N.et al. Delivery of anti-inflammatory nutraceuticals by nanoparticles for the prevention and treatment of cancer. Biochemical Pharmacology, In Press, Uncorrected Proof, 2010.

HERGT, R.et al. Physical limits of hyperthermia using magnetite fine particles. IEEE Transactions on Magnetism, 34, p. 3745-3754, 1998.

ITO, A.et al. Time course of biodistribution and heat generation of magnetic cationic lipossomes in mouse model. Japanese Journal of Hyperthermic Oncology, 19, p. 151-159, 2003.

JOHNSON, G. A.et al. Histology by magnetic resonance microscopy. Magnetic Resonance, Q 9, p. 1-30, 1993.

JÓZEFCZAK, A.; SKUMIEL, A. Study of heating effect and acoustic properties of dextran stabilized magnetic fluid. Journal of Magnetism and Magnetic Materials, 311, p. 193-196, 2007.

KAWAGUCHI, H. Functional polymer microspheres. Progress in Polymer Science, 25, p. 1171-1210, 2000.

KNOBEL, M. Partículas finas: superparamagnetismo e magnetorresistência gigante. Revista Brasileira do Ensino de Física, v. 22, n. 3, 2000.

KNOBEL, M.; GOYA, G. F. Ferramentas magnéticas na escala do átomo. Scientific American Brasil, 31, p. 58-66, 2004.

LAWACZECK, R.et al. Magnetic iron oxide particles coated with carboxydextran for parental administration and liver contrasting. Acta Radiologica, 38, p. 584-597, 1997.

LI, Z.et al. Magnetite nanoparticles with high heating efficiencies for application in the hyperthermia of cancer. Materials Science and Engineering C, 30, p. 990-996, 2010.

LÜBBE, A. S.; ALEXIOU, C.; BERGEMANN, C. Clinical applications of magnetic drug targeting. Journal of Surgical Research, 95, p. 200-206, 2001.

MAGENHEIN, B.; BENITA, S. Nanoparticle characterization: a comprehensive physicochemical approach. STP Pharma Sciences, 1, p. 221-241, 1991.

MICHEL, S. C. A.et al. Preoperative breast cancer staging: MR imaging of the axilla with ultrasmall superparamagnetic iron oxide enhancement. Radiology, 225, p. 527-536, 2002. 
MITSUMORI, M.et al. Targeted hyperthermia using dextran magnetite complex: a new treatment modality for liver tumors. Hepatogastroenterology, 43, p. 1431-1437, 1996.

MITSUMORI, M.et al. Time course of the heat-generating capability of dextran magnetite complex (DM) in vivo. Japanese Journal of Hyperthermic Oncology, 17, p. 85-91, 2001.

MRINMOY, D.; GHOSH, P. S.; ROTELLO, V. M. Applications of nanoparticles in biology. Advanced Materials, 20, p. 4225-4241, 2008.

MITANI, S.; FUJIMORI, H.; OHNUMA, S. Temperature dependence of tunnel-type GMR in insulating granular systems. Journal of Magnetism and Magnetic Materials, 171-181, p. 919-920, 1998.

NANZ, D.et al. TE-Switched Double-Contrast Enhanced Visualization of Vascular System and Instrumentals for MR-Guided. Magnetic Resonance in Medicine, 43, p. 645-648, 2000.

NÉEL, L. Nouvelle théorie du champ coercitif. Physica, 15, p. 225-234, 1949.

OLIVEIRA, A. G.et al. Microemulsões: estrutura e aplicações como sistema de liberação de fármacos. Química Nova, 27, p. 131-138, 2004.

PANKHURST, Q. A.et al. Applications of magnetic nanoparticles in biomedicine. Journal of Physics D: Applied Physics, 36, p. R167-R181, 2003.

PAKHOMOV, A. B.; YAN, X.; ZHAO, B. Giant Hall effect in percolating ferromagnetic granular metal-insulator films. Applied Physics Letters, 67, p. 3497-3499, 1995.

PELECKY, D. L.; RIEKE, R. D. Magnetic properties of nanostructured materials. Chemical Mater, 8, p. 1770-1783, 1996.

POULIQUEN, D.et al. Superparamagnetic iron oxide nanoparticles as a liver MRI contrast agent: contribution of microencapsulation to improved redistribution. Magnetic Resonance Imaging, v.7, n. 7, 1989.

PUISIEUX, F.; ROBLOT-TREPEL, L. Vetorização e vetores de fármacos. Caderno de Farmácia, 4, p. 29-50, 1988.

ROSENSWEIG, R. E. Heating magnetic fluid with alternating magnetic field. Journal of Magnetism and Magnetic Materials, 252, p. 370-374, 2002.

RUEHM, S. G.et al. Magnetic resonance imaging of atherosclerotic plaque with ultrasmall superparamagnetic particles of iron oxides in hyperlipidemic rabbits. Circulation, 103, p. 415-422, 2001.

SAINI, S.et al. Particulate iron oxide (magnetite): a reticuloendothelial system specific liver MRI contrast agent. Magnetic Resonance Imaging, v.4, n.2, 1986.

SAINI, S.et al. Multicentre Dose-Ranging Study on the Efficacy of USPIO Ferumoxtran-10 for Liver MR Imaging. Clinical Radiology, 55, p. 690-695, 2000. 
SCHÜTT, W.et al. New methods for investigation of blood biomaterial interaction. Journal of Artificial Organs, 19, p. 847-851, 1995.

SEMELKA, R. C.; HELMBERGER, T. K. G. Contrast agents for MR imaging of the liver. Radiology, 218, p. 27-38, 2001.

SHINKAI, M.; MATSUI, M.; KOBAYASHI, T. Heat properties of magnetolipossomes for local hypertermia. Japanese Journal of Hiperthermic Oncology, 10, p. 168-177, 1994.

SHUBAYEV, V. I.; PISANIC II, T. R.; JIN. S. Magnetic nanoparticles for theragnostics. Advanced Drug Delivery Reviews, 61, p. 467-477, 2009.

SILVA, H. S. R. C.; SANTOS, K. S. C. R.; FERREIRA, E. I. Quitosana: derivados hidrossolúveis, aplicações farmacêuticas e avanços. Química Nova, v.29, n.4, p. 776-785, 2006.

SKOMSKI, R. Nanomagnetics. Journal of Physics: Condensed Matter, 15, p. R841-R896, 2003.

SPEISER, P. P. Nanoparticles and lipossomes: a state of the art. Methods and Findings in Experimental and Clinical Pharmacology, 13, p. 337-342, 1991.

TARTAJ, P.et al. The preparation of magnetic nanoparticles for applications in biomedicine. Journal of Physics D: Applied Physics, 36, p. R182-R197, 2003.

WACKER, F. K.et al. MR image-guided endovascular procedures with the ultrasmall superparamagnetic iron oxide SHU555C as an intravascular contrast agent: study in pigs. Radiology, 226, p. 459-464, 2003.

WEISSLEDER, R.et al. Ultrasmall superparamagnetic iron oxide: characterization of a new class of contrast agents for MR imaging. Radiology, 175, p. 489-493, 1990.

XIAO, J. Q.; JIANG, J. S.; CHIEN, C. L. Giant magnetoresistive properties in granular transition metals. IEEE Transactions on Magnetics, 29, p. 2688-2693, 1993.

YANASE, M.et al. Intracellular hyperthermia for cancer using magnetite cationic lipossomes: an in vivo study. Japanese Journal of Cancer Research, 89, p. 463-469, 1998.

ZHANG, X.; WEBB, A. Design of a four-coil surface array for in vivo magnetic resonance microscopy at $600 \mathrm{MHz}$. Concepts in Magnetic Resonance Part B: Magnetic Resonance Engineering, 24B, p. 6-14, 2005. 
